\title{
Reduced-Complexity Semidefinite Relaxations of Optimal Power Flow Problems
}

\author{
Martin S. Andersen, Anders Hansson and Lieven Vandenberghe
}

\author{
Linköping University Post Print
}

Tweet

N.B.: When citing this work, cite the original article.

(C)2009 IEEE. Personal use of this material is permitted. However, permission to reprint/republish this material for advertising or promotional purposes or for creating new collective works for resale or redistribution to servers or lists, or to reuse any copyrighted component of this work in other works must be obtained from the IEEE.

Martin S. Andersen, Anders Hansson and Lieven Vandenberghe, Reduced-Complexity Semidefinite Relaxations of Optimal Power Flow Problems, 2014, IEEE Transactions on Power Systems, (29), 4, 1855-1863.

http://dx.doi.org/10.1109/TPWRS.2013.2294479

Postprint available at: Linköping University Electronic Press

http://urn.kb.se/resolve?urn=urn:nbn:se:liu:diva-109172 


\title{
Reduced-Complexity Semidefinite Relaxations of Optimal Power Flow Problems
}

\author{
Martin S. Andersen, Anders Hansson, Senior member, IEEE, Lieven Vandenberghe, Senior member, IEEE
}

\begin{abstract}
We propose a new method for generating semidefinite relaxations of optimal power flow problems. The method is based on chordal conversion techniques: by dropping some equality constraints in the conversion, we obtain semidefinite relaxations that are computationally cheaper, but potentially weaker, than the standard semidefinite relaxation. Our numerical results show that the new relaxations often produce the same results as the standard semidefinite relaxation, but at a lower computational cost.
\end{abstract}

Index Terms-optimal power flow, semidefinite relaxation, chordal conversion

\section{INTRODUCTION}

The general AC optimal power flow (OPF) problem is an extension of the economic dispatch problem introduced by Carpentier in 1962 [1]. The goal of the OPF problem is to find a cost-optimal operating-point of a power system that consists of a set of power busses that are interconnected through a network of transmission lines. Each power bus may have one or more generators and/or a load (i.e., demand), and typical problem formulations minimize generation cost or transmission loss, subject to a set of nonlinear power flow constraints and bounds on e.g. generation, voltage magnitudes, and transmission line flows. Many different problem formulations exist, but AC OPF problems are generally difficult nonconvex optimization problems; see e.g. [2], [3] for further details on different formulations and applications.

Numerous solution techniques have been proposed in previous work, including nonlinear optimization techniques such as sequential quadratic programming, Lagrangian relaxation, and interior-point methods, and more recently, derivative-free methods such as particle swarm optimization, genetic algorithms, and evolutionary programming; see e.g. [4], [5] and references therein. Although the derivative-free methods are generally more versatile, the conventional nonlinear optimization techniques have some important advantages. For example, derivative-free methods do not compute dual variables which have valuable economic meanings in electricity markets.

Martin S. Andersen is with the Department of Applied Mathematics and Computer Science, Technical University of Denmark, 2800 Lyngby, Denmark (e-mail: mskan@dtu.dk). Part of this research was conducted while he was a postdoc at Linköping University. Research supported by ERC Grant ERC2011-AdG-20110209.

Anders Hansson is with the Division of Automatic Control, Department of Electrical Engineering, Linköping University, SE-581 83 Linköping, Sweden (e-mail: anders.g.hansson@liu.se). Research supported by the Swedish Department of Education within the ELLIIT project.

Lieven Vandenberghe is with the Electrical Engineering Department, University of California, Los Angeles, 66-147L Engineering IV, CA 90095-1594 (e-mail: vandenbe@ee.ucla.edu). Research supported by NSF Grants DMS1115963 and ECCS-1128817.
In recent work, researchers have applied semidefinite relaxation (SDR) techniques to various OPF problem formulations [6]-[8]. This approach leads to conic optimization problems that can be solved in polynomial time with an interiorpoint method. The SDR formulation has attracted significant attention since, unlike previous methods, the solution provides either (i) a certificate of global optimality, (ii) a certificate of infeasibility, or (iii) a lower bound on the optimal value. We say that the SDR is "exact" if it provides the global solution to the original problem. Conditions that guarantee exactness of the SDR have been derived and studied in [8]-[10].

The computational cost of solving the SDR problem grows rapidly with the size of the OPF problem, and this renders the direct SDR formulation impractical for large-scale OPF problems. This can be attributed to two computational bottlenecks: (i) a dense symmetric matrix variable that grows with the number of busses in the power network, and (ii) a large dense positive definite system of equations, the so-called Schur complement system, that defines the search direction at each iteration. Despite its apparent high computational complexity, the SDR formulation possesses both sparsity and low-rank structure. The sparsity is related to the fact that each power bus is typically connected to only a small number of adjacent power busses, and, in fact, the dual variable inherits its sparsity pattern from the network graph. This was pointed out by Lavaei \& Low [8] who proposed to solve the dual problem; however, they solve the dual problem with SeDuMi [11], which is a general-purpose primal-dual conic solver. The solver therefore maintains and factors at each iteration not only the sparse dual variable, but also the dense primal variable. It is important to note that the second computational bottleneck (the dense Schur complement system) remains even if one avoids forming the dense primal variable, for example, by solving the SDR problem and/or its dual using a sparse semidefinite programming (SDP) solver such as DSDP [12] or SMCP [13].

Inspired by advances in sparse semidefinite programming, Jabr [14] applied the conversion method by Fukuda et al. [15] to the SDR of the OPF problem. The conversion method is based on positive semidefinite matrix completion, and it reformulates a sparse SDP as an equivalent block-diagonal SDP with additional equality constraints. When applied to a tree network, the conversion method yields a block-diagonal SDP with $2 \times 2$ blocks. In general, the blocks correspond to the so-called cliques of a chordal embedding of the network graph, and the extra equality constraints impose consistency by forcing certain elements of different blocks to be equal. Although this method typically introduces sparsity in the Schur complement system, it also increases the order of the Schur 
complement system quite substantially in many cases. The benefit of using the conversion method therefore depends very much on the network graph and on the chordal embedding. In [16], Molzahn et al. apply the conversion method in combination with a simple heuristic for reducing the number of cliques in the converted problem, and their method typically reduces the computation time by a factor of three. However, like previous work in this area, the conversion is performed based on the SDR of a real-valued formulation of the problem, and as we explain in Section IV, this approach adds more than twice as many equality constraints to the converted problem than necessary (unless the special structure of the real-valued problem is taken into account).

a) Contributions: The main goal of this paper is to propose new formulations and relaxations of the AC OPF problem. Specifically, we propose a class of new computationally cheaper SDRs of the OPF problem which are obtained by dropping some of the equality constraints introduced when converting the original SDR to a block-diagonal one. We also propose a primal formulation of the $\mathrm{AC} \mathrm{OPF}$ problem in which we model line flow constraints using second-order cones (SOCs) of dimension three instead of positive semidefinite cones of order three. This reduces the number of variables. Furthermore, by using complex-valued voltage variables in our model instead of their real-valued real and imaginary parts, we obtain an SDR that involves a Hermitian matrix variable of order equal to the number of power busses instead of a symmetric matrix variable of twice the order. By applying the conversion method of Fukuda et al. [15] to this SDR, we avoid introducing unnecessary equality constraints.

b) Notation: We denote by $\mathbb{R}^{n}$ and $\mathbb{C}^{n}$ the sets of real and complex $n$-dimensional vectors, and $\mathbb{R}^{m \times n}$ and $\mathbb{C}^{m \times n}$ denote the sets of real and complex $m \times n$ matrices. The set $\mathcal{H}^{n}$ is the set of Hermitian matrices of order $n$, and $\mathcal{H}_{+}^{n}$ denotes the set of positive semidefinite matrices in $\mathcal{H}^{n}$. The matrix inequality $A \succeq B$ means that $A-B$ is positive semidefinite, i.e., the eigenvalues of $A-B$ are nonnegative. We denote by $\jmath=\sqrt{-1}$ is the imaginary unit, $a^{*}$ denotes the complex conjugate of $a \in \mathbb{C}$, and $A^{H}$ denotes the Hermitian transpose of a matrix $A \in \mathbb{C}^{m \times n}$. Finally, $\operatorname{tr}(A)$ denotes the trace of a square matrix $A$, and $\operatorname{tr}\left(B^{H} A\right)$ denotes the inner product of $A$ and $B$.

c) Outline: The rest of the paper is organized as follows. We first introduce the power system model and our formulation of the OPF problem and its SDR in Section II. We then review chordal conversion in Section III and introduce some new SDRs in Section IV. This is followed by numerical experiments in Section V, and we conclude the paper in Section VI.

\section{Problem Formulation and Semidefinite RELAXATION}

We start this section by describing the power system model and a variant of the AC OPF problem. We then propose a reformulation of the problem and derive the associated SDR.

\section{A. Model}

The power system model consists of a network of power busses. We denote the set of power busses (nodes) by $\mathcal{N}$ and the set of transmission lines (edges) by $\mathcal{L} \subseteq \mathcal{N} \times \mathcal{N}$, i.e., $(k, l) \in \mathcal{L}$ if there is a transmission line from node $k$ to node $l$. Transmission lines may be nonsymmetric and there may be more than one transmission line between a pair of nodes, so we model the network graph as a directed graph. We denote the number of nodes by $|\mathcal{N}|$ and the number of transmission lines by $|\mathcal{L}|$. For each $k \in \mathcal{N}$, we define $\mathcal{G}_{k}$ as the set of generators at node $k$, and $\mathcal{G}=\bigcup_{k \in \mathcal{N}} \mathcal{G}_{k}$ denotes the set of all generators in the network. We allow $\mathcal{G}_{k}$ to be the empty set which corresponds to a power bus without generators. We also define a set $\mathcal{F} \subseteq \mathcal{L}$ of transmission lines with flow constraints (i.e., $\mathcal{F}=\mathcal{L}$ if all transmission lines have flow constraints).

We associate with power bus $k$ a complex bus voltage $v_{k}$ and a complex current $i_{k}$, and we define two vectors $v=\left(v_{1}, v_{2}, \ldots, v_{n}\right)$ and $i=\left(i_{1}, i_{2}, \ldots, i_{n}\right)$. The currents and voltages satisfy the equation $i=Y v$ where $Y \in \mathbb{C}^{n \times n}$ is a so-called bus admittance matrix which inherits its sparsity from the network graph, thus $Y$ is generally very sparse. We will henceforth assume that the network graph is connected. The bus admittance matrix follows from Kirchhoff's current law, and it can be computed from the problem data; refer to e.g. [17] for details on how to compute the bus admittance matrix. We denote the complex power generated by generator $g$ by $s_{g}=p_{g}+\jmath q_{g}$, and similarly, $S_{k}^{\mathrm{d}}=P_{k}^{\mathrm{d}}+\jmath Q_{k}^{\mathrm{d}}$ denotes the known demand or load at bus $k$.

The OPF problem takes the following form

$$
\text { minimize } \sum_{g \in \mathcal{G}} f_{g}\left(p_{g}\right)
$$

subject to $i=Y v$ and the constraints

$$
\begin{gathered}
i_{k}^{*} v_{k}=\sum_{g \in \mathcal{G}_{k}} s_{g}-S_{k}^{\mathrm{d}}, \quad k \in \mathcal{N} \\
P_{g}^{\min } \leq p_{g} \leq P_{g}^{\max }, \quad g \in \mathcal{G} \\
Q_{g}^{\min } \leq q_{g} \leq Q_{g}^{\max }, \quad g \in \mathcal{G} \\
V_{k}^{\min } \leq\left|v_{k}\right| \leq V_{k}^{\max }, \quad k \in \mathcal{N} \\
\left|S_{k, l}^{\mathrm{fl}}(v)\right| \leq S_{k, l}^{\max }, \quad(k, l) \in \mathcal{F} \\
\left|S_{l, k}^{\mathrm{fl}}(v)\right| \leq S_{k, l}^{\max }, \quad(k, l) \in \mathcal{F} .
\end{gathered}
$$

The constraints (1b) are the power balance equations, (1c)(1d) are real and reactive power generation constraints, (1e) are voltage magnitude constraints, and (1f)-(1g) are constraints on transmission line flows. The variables are $i, v$, and $s_{g}=$ $p_{g}+\jmath q_{g}$ for $g \in \mathcal{G}$, and $S_{k, l}^{\mathrm{fl}}(v)$ denotes the complex power flow from bus $k$ to bus $l$ which is a quadratic function of $v_{k}$ and $v_{l}$. We will use the notation $S_{k, l}^{\mathrm{fl}}(v)=v^{H} T_{k, l} v+$ $\jmath^{H} \widetilde{T}_{k, l} v$ where $T_{k, l}$ and $\widetilde{T}_{k, l}$ are Hermitian and given (see [17] for details regarding the transmission line model). The scalars $P_{g}^{\min }, P_{g}^{\max }, Q_{g}^{\min }, Q_{g}^{\max }, V_{k}^{\min }, V_{k}^{\max }$, and $S_{k, l}^{\max }$ are real and given, and the cost function $f_{g}: \mathbb{R} \rightarrow \mathbb{R}$ represents the fuel cost model of generator $g$ and can be any so-called semidefinite representable convex function. This includes the set of linear and convex quadratic representable functions; see 
e.g. [18]. Here we will model the fuel cost curve as a convex quadratic function of the form

$$
f_{g}\left(p_{g}\right)=\alpha_{g} p_{g}^{2}+\beta_{g} p_{g}
$$

where the scalars $\alpha_{g} \geq 0$ and $\beta_{g}$ are given; see e.g. [2].

\section{B. Reformulation and Semidefinite Relaxation}

If we denote by $e_{k}$ the $k$ th column of the identity matrix of order $n$, we can express the left-hand side of each of the power balance equations (1b) as $i_{k}^{*} v_{k}=v^{H} Y^{H} e_{k} e_{k}^{T} v$, and hence we can eliminate the variables $i_{k}$ and remove the equation $i=Y v$ from (1). The real power generation inequalities (1c) can be expressed as

$$
p_{g}=P_{g}^{\min }+p_{g}^{l}, \quad p_{g}^{l}+p_{g}^{u}=P_{g}^{\max }-P_{g}^{\min },
$$

where $p_{g}^{l}$ and $p_{g}^{u}$ are nonnegative slack variables, and the equation $p_{g}=P_{g}^{\min }+p_{g}^{l}$ allows us to eliminate the free variable $p_{g}$. In a similar fashion, we may introduce nonnegative slack variables $q_{g}^{l}$ and $q_{g}^{u}$ for each of the inequalities (1d), and each of the voltage constraints (1e) can be expressed as

$$
V_{k}^{\min }+\nu_{k}^{l}=\left|v_{k}\right|, \quad\left|v_{k}\right|+\nu_{k}^{u}=V_{k}^{\max },
$$

with nonnegative slack variables $\nu_{k}^{l}$ and $\nu_{k}^{u}$. We now define two Hermitian matrices $Y_{k}=(1 / 2)\left(Y^{H} e_{k} e_{k}^{T}+e_{k} e_{k}^{T} Y\right)$ and $\widetilde{Y}_{k}=-(\jmath / 2)\left(Y^{H} e_{k} e_{k}^{T}-e_{k} e_{k}^{T} Y\right)$ so that the real and reactive power balance equations at bus $k$ can be expressed as

$$
\begin{aligned}
& \sum_{g \in \mathcal{G}_{k}}\left(P_{g}^{\mathrm{min}}+p_{g}^{l}\right)=v^{H} Y_{k} v+P_{k}^{\mathrm{d}}, \\
& \sum_{g \in \mathcal{G}_{k}}\left(Q_{g}^{\mathrm{min}}+q_{g}^{l}\right)=v^{H} \widetilde{Y}_{k} v+Q_{k}^{\mathrm{d}} .
\end{aligned}
$$

The convex quadratic cost functions (2) can be expressed using the epigraph formulation $f_{g}\left(p_{g}\right) \leq t_{g}$ where $t_{g}$ is an auxiliary variable, and hence the inequality can be expressed as the quadratic constraint $\left|\sqrt{\alpha_{g}} p_{g}\right|^{2} \leq t_{g}-\beta_{g} p_{g}$. This, in turn, is equivalent to a SOC constraint (see e.g. [18])

$$
\left\|\left[\begin{array}{c}
1 / 2-t_{g}+\beta_{g} p_{g} \\
\sqrt{2 \alpha_{g}} p_{g}
\end{array}\right]\right\|_{2} \leq 1 / 2+t_{g}-\beta_{g} p_{g} .
$$

Using the above reformulations and slack variables, and if we partition $\mathcal{G}$ into a set of generators $G^{\text {lin }}=\left\{g \in \mathcal{G} \mid \alpha_{g}=0\right\}$ with linear cost and generators $G^{\text {quad }}=\left\{g \in \mathcal{G} \mid \alpha_{g}>0\right\}$ with quadratic cost, the OPF problem (1) can be expressed as

$$
\operatorname{minimize} \sum_{g \in \mathcal{G}^{\text {lin }}} \beta_{g}\left(P_{g}^{\text {min }}+p_{g}^{l}\right)+\sum_{g \in \mathcal{G}^{\text {quad }}} t_{g}
$$

subject to

$$
\begin{gathered}
\operatorname{tr}\left(Y_{k} X\right)=\sum_{g \in \mathcal{G}_{k}}\left(P_{g}^{\min }+p_{g}^{l}\right)-P_{k}^{\mathrm{d}}, k \in \mathcal{N} \\
\operatorname{tr}\left(\widetilde{Y}_{k} X\right)=\sum_{g \in \mathcal{G}_{k}}\left(Q_{g}^{\min }+q_{g}^{l}\right)-Q_{k}^{\mathrm{d}}, k \in \mathcal{N} \\
p_{g}^{l}+p_{g}^{u}=P_{g}^{\max }-P_{g}^{\min }, g \in \mathcal{G} \\
q_{g}^{l}+q_{g}^{u}=Q_{g}^{\max }-Q_{g}^{\min }, g \in \mathcal{G} \\
\left(V_{k}^{\min }\right)^{2}+\nu_{k}^{l}=X_{k k}, X_{k k}+\nu_{k}^{u}=\left(V_{k}^{\max }\right)^{2}, k \in \mathcal{N}
\end{gathered}
$$

$$
\begin{gathered}
z_{k, l}=\left[\begin{array}{c}
\left(S_{k, l}^{\max }\right) \\
\operatorname{tr}\left(T_{k, l} X\right) \\
\operatorname{tr}\left(\widetilde{T}_{k, l} X\right)
\end{array}\right], z_{l, k}=\left[\begin{array}{c}
\left(S_{k, l}^{\max }\right) \\
\operatorname{tr}\left(T_{l, k} X\right) \\
\operatorname{tr}\left(\widetilde{T}_{l, k} X\right)
\end{array}\right],(k, l) \in \mathcal{F} \\
w_{g}=\left[\begin{array}{c}
1 / 2+t_{g}-\beta_{g}\left(P_{g}^{\min }+p_{g}^{l}\right) \\
1 / 2-t_{g}+\beta_{g}\left(P_{g}^{\min }+p_{g}^{l}\right) \\
\sqrt{2 \alpha_{g}}\left(P_{g}^{\min }+p_{g}^{l}\right)
\end{array}\right], g \in \mathcal{G}^{\text {quad }} \\
p_{g}^{l}, p_{g}^{u}, q_{g}^{l}, q_{g}^{u} \geq 0, g \in \mathcal{G} \\
\nu_{k}^{l}, \nu_{k}^{u} \geq 0, k \in \mathcal{N} \\
z_{k, l} \in \mathcal{K}_{q}^{3}, z_{l, k} \in \mathcal{K}_{q}^{3},(k, l) \in \mathcal{F} \\
w_{g} \in \mathcal{K}_{q}^{3}, g \in \mathcal{G}^{\text {quad }} \\
X=v v^{H} .
\end{gathered}
$$

Here $\mathcal{K}_{q}^{3}=\left\{(t, x) \in \mathbb{R} \times \mathbb{R}^{2} \mid t \geq\|x\|\right\}$ denotes the SOC in $\mathbb{R}^{3}$. The constraints $(3 \mathrm{~g})$ and $(3 \mathrm{k})$ correspond to the line flow constraints (1f)-(1g), and the constraints (3h) and (3l) correspond to the epigraph formulation of the cost functions for generators with quadratic cost.

The only nonconvex constraint in (3) is the rank-1 constraint (3m), and we obtain an SDR of the problem simply by replacing the nonconvex constraint $X=v v^{H}$ with the positive semidefinite constraint $X \succeq 0$. The SDR problem is convex, and its solution $X^{\star}$ provides a lower bound on the optimal value of (1). Furthermore, if $X^{\star}$ has rank 1, we obtain a globally optimal solution to (1) by computing a rank-1 factorization $X^{\star}=\tilde{v} \tilde{v}^{H}$. Note that $X^{\star}$ only carries relative phase information since $\tilde{v} \tilde{v}^{H}=\bar{v} \bar{v}^{H}$ for any $\bar{v}=\exp (\jmath \theta) \tilde{v}$ where $\theta \in[0,2 \pi]$.

The SDR of (3) can be expressed as a "cone linear program" (cone LP) of the form

$$
\begin{array}{ll}
\operatorname{minimize} & h^{T} z \\
\text { subject to } & G^{T} z+c=0 \\
& z \in \mathcal{K}
\end{array}
$$

with variable $z$ and where $\mathcal{K}$ is the direct product of cones. To see this, notice that the constraints (3b)-(3h) are linear equality constraints. There are a total of

$$
r=4|\mathcal{N}|+2|\mathcal{G}|+3\left(2|\mathcal{F}|+\left|\mathcal{G}^{\text {quad }}\right|\right)
$$

of these, and they correspond to the constraint $G^{T} z+c=0$ in (4). Similarly, the cone constraint $z \in \mathcal{K}$ in (4) corresponds to the $4|\mathcal{G}|+2|\mathcal{N}|$ nonnegativity constraints (3i)-(3j), the $2|\mathcal{F}|+$ $\left|\mathcal{G}^{\text {quad }}\right|$ SOC constraints (3k)-(3l), and the constraint $X \succeq 0$ (i.e., the relaxed version of (3m)). In other words, $z$ represents all variables in the SDR of (3), and the cone $\mathcal{K}$ is given by

$$
\mathcal{K}=\mathbb{R}_{+}^{4|\mathcal{G}|+2|\mathcal{N}|} \times \mathcal{K}_{q} \times \mathcal{H}_{+}^{|\mathcal{N}|}
$$

where $\mathcal{K}_{q}=\mathcal{K}_{q}^{3} \times \cdots \times \mathcal{K}_{q}^{3}$ is the direct product of $2|\mathcal{F}|+$ $\left|\mathcal{G}^{\text {quad }}\right|$ SOCs. Thus, the total number of variables is equal to

$$
4|\mathcal{G}|+2|\mathcal{N}|+3\left(2|\mathcal{F}|+\left|\mathcal{G}^{\text {quad }}\right|\right)+|\mathcal{N}|^{2}
$$

where $|\mathcal{N}|^{2}$ is the number of scalar variables needed to represent the Hermitian matrix variable $X$.

Before we present our new relaxations of the OPF problem, we note that the problem in (3) is equivalent to a nonconvex quadratic optimization problem, and hence it is also possible to derive relaxations based on linear optimization and SOC 


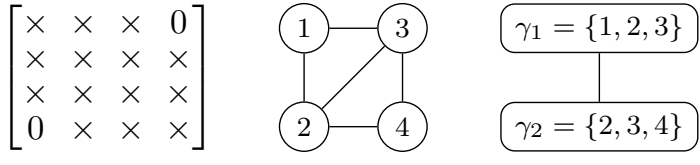

Fig. 1. Example of sparsity pattern, sparsity graph, and clique tree.

optimization; see e.g. [19] and references therein. While these relaxations are computationally more tractable than the SDR of (3), they are also weaker in general, which means that they may provide more conservative lower bounds than the standard SDR. For example, it is possible to obtain a SOC relaxation of (3) simply by replacing the constraint $X \succeq 0$ in the SDR of (3) by positive semidefiniteness constraints on some or all $2 \times 2$ principal minors of $X$. These constraints can be expressed as SOC constraints since if $W$ is Hermitian and of order 2, then

$$
W=\left[\begin{array}{ll}
x & y^{*} \\
y & w
\end{array}\right] \succeq 0 \Leftrightarrow\left\|\left[\begin{array}{c}
x-w \\
2 \Re y \\
2 \Im y
\end{array}\right]\right\|_{2} \leq x+w .
$$

It is important to note that the positive semidefiniteness of the $2 \times 2$ principal minors of $X$ is only a necessary condition, but not a sufficient condition, for positive semidefiniteness of $X$, so a SOC relaxation is generally weaker than the SDR.

\section{ChORDAl CONVERSION}

We begin this section with a quick review of chordal sparsity and chordal cones; a more comprehensive treatment of these concepts can be found in e.g. [20]-[22].

\section{A. Chordal Sparsity Patterns and Cones}

We represent a symmetric sparsity pattern of order $n$ as a set of index pairs $E \subseteq\{1,2, \ldots, n\} \times\{1,2, \ldots, n\}$ where each pair $(i, j) \in E$ corresponds to a nonzero entry of a sparse matrix of order $n$. We associate with the sparsity pattern $E$ an undirected graph which has $n$ nodes $\{1,2, \ldots, n\}$ and an edge between nodes $i$ and $j(i \neq j)$ if $(i, j) \in E$. Fig. 1 shows an example of a sparsity pattern and the associated sparsity graph. We say that the sparsity pattern $E$ is chordal if the sparsity graph is chordal. A graph is chordal if all cycles of length greater than or equal to 4 have a chord (i.e., an edge joining two non-adjacent nodes in a cycle). Note that an immediate consequence of this definition is that acyclic graphs are chordal. A clique of the sparsity graph is a node subset $\gamma \subseteq\{1,2, \ldots, n\}$ such that the subgraph induced by $\gamma$ is complete and maximal. Here "maximal" means that there exists no other complete subgraph that properly contains the subgraph induced by $\gamma$. In other words, each clique of the sparsity graph corresponds to a maximal dense principal submatrix of the sparsity pattern $E$. It is easy to verify that the sparsity graph in Fig. 1 is chordal (there are no cycles of length 4 without a chord), and there are two cliques $\gamma_{1}=\{1,2,3\}$ and $\gamma_{2}=\{2,3,4\}$. Indeed, these index sets correspond to maximal dense principal submatrices in the sparsity pattern. Note that the set $\{1,2\}$ also induces a complete subgraph, but is is not maximal since it is contained in $\gamma_{1}$, and hence it is not a clique according to our definition. As a final example, we mention that a clique of a connected acyclic graph is any pair of nodes that are connected by an edge, and this implies that a connected acyclic graph with $n$ nodes has $n-1$ cliques of order 2 .

We denote by $\mathcal{E}_{\gamma}(X)$ the principal submatrix $X_{\gamma, \gamma}$ of $X$ defined by the index set $\gamma$. Similarly, we denote the adjoint operator by $\mathcal{E}_{\gamma}^{\operatorname{adj}}(W)$ which takes a matrix $W$ of order $|\gamma|$ and returns a matrix of order $n$ with $W$ in the principal submatrix defined by $\gamma$ and with zeros elsewhere.

Suppose $\gamma_{1}, \gamma_{2}, \ldots, \gamma_{m}$ are the cliques of a connected chordal graph. (We have $m<n$ since a connected chordal graph with $n$ vertices has at most $n-1$ cliques.) The cliques can be arranged in a clique tree (a maximum weight spanning tree of the clique intersection graph) that satisfies the so-called running intersection property, i.e., $\gamma_{i} \cap \gamma_{j} \subseteq \gamma_{k}$ if clique $k$ is on the path between cliques $i$ and $j$ in the tree; see e.g. [20]. Given a chordal graph, the cliques and a clique tree can be found efficiently using e.g. the algorithm by Pothen $\&$ Sun [23]. Nonchordal graphs can be handled by means of a chordal embedding, and this technique is closely related to sparse symbolic factorization techniques; see e.g. [24] and references therein. In particular, a chordal embedding can be found using a fill reducing reordering (such as "approximate minimum degree" or "nested dissection") in combination with a symbolic Cholesky factorization.

Given a sparsity pattern $E$, we define $\mathcal{H}_{E}^{n}$ as the set of Hermitian matrices of order $n$ and with sparsity pattern $E$, and $\mathcal{P}_{E}(X)$ denotes the projection of a (possibly dense) Hermitian matrix $X$ onto $\mathcal{H}_{E}^{n}$. We define the cone of positive semidefinite completable matrices in $\mathcal{H}_{E}^{n}$ as $\mathcal{H}_{E, \mathrm{c}}^{n}=\left\{\mathcal{P}_{E}(X) \mid X \succeq 0\right\}$. A key result by Grone et al. [25, Theorem 7] establishes that the cone $\mathcal{H}_{E, \mathrm{c}}^{n}$ is equivalent to the set of partial positive semidefinite matrices in $\mathcal{H}_{E}^{n}$ (i.e., matrices in $\mathcal{H}_{E}^{n}$ for which all dense principal submatrices in $E$ are positive semidefinite) if and only if the sparsity pattern $E$ is chordal.

\section{B. The Conversion Method}

The conversion method of Fukuda et al. [15] (which was further studied and generalized by Kim et al. in [26]) makes use of the aforementioned result by Grone et al. to express a cone constraint $X \in \mathcal{H}_{E, \mathrm{c}}^{n}$ (where $E$ is henceforth assumed to be chordal) as $m$ coupled constraints

$$
W_{k} \succeq 0, W_{k}=\mathcal{E}_{\gamma_{k}}(X), k=1, \ldots, m,
$$

with $X \in \mathcal{H}_{E}^{n}$ and $W_{k} \in \mathcal{H}^{\left|\gamma_{k}\right|}$. Using the running intersection property, we can eliminate the variable $X$ in (5), i.e., $W_{k}=$ $\mathcal{E}_{\gamma_{k}}(X)$ for some $X \in \mathcal{H}_{E, \mathrm{c}}^{n}$ if and only if for $k=1, \ldots, m$,

$$
W_{k} \succeq 0, \mathcal{E}_{\gamma_{j} \cap \gamma_{k}}\left(\mathcal{E}_{\gamma_{j}}^{\operatorname{adj}}\left(W_{j}\right)-\mathcal{E}_{\gamma_{k}}^{\operatorname{adj}}\left(W_{k}\right)\right)=0, j \in \operatorname{ch}(k)
$$

where $\operatorname{ch}(k)$ is the set of indices of the cliques in the clique tree that are children of clique $k$ (node $j$ is a child of node $k$ in a rooted tree if there is an edge between $j$ and $k$, and $k$ is on the path between $j$ and the root of the tree). Note that the constraint $\mathcal{E}_{\gamma_{j} \cap \gamma_{k}}\left(\mathcal{E}_{\gamma_{j}}^{\operatorname{adj}}\left(W_{j}\right)-\mathcal{E}_{\gamma_{k}}^{\operatorname{adj}}\left(W_{k}\right)\right)=0$ in (6) couples principal submatrices of $W_{j}$ and $W_{k}$. These submatrices are of order $\left|\eta_{j}\right|$ where $\eta_{j}=\gamma_{j} \cap \gamma_{k}$, and since $W_{k}$ and $W_{j}$ are both Hermitian, the coupling consists of a total of $\left|\eta_{j}\right|^{2}$ real 
equality constraints, i.e., $\left|\eta_{j}\right|\left(\left|\eta_{j}\right|+1\right) / 2$ equality constraints that correspond to the symmetric real part and $\left|\eta_{j}\right|\left(\left|\eta_{j}\right|-1\right) / 2$ equality constraints that correspond to the skew-symmetric imaginary part. The conversion (6) therefore introduces a total of $s=\sum_{k=1}^{m} \sum_{j \in \operatorname{ch}(k)}\left|\eta_{j}\right|^{2}$ equality constraints. The Hermitian matrix inequality $X \succeq 0$ can also be expressed as the real-valued symmetric matrix inequality

$$
Z=\left[\begin{array}{cc}
\Re X & -\Im X \\
\Im X & \Re X
\end{array}\right] \succeq 0
$$

and if we apply the conversion method to $Z$ without exploiting its particular structure, as has been done in previous work (e.g., [14], [16]), we add unnecessary consistency constraints. Specifically, applying the conversion method directly to $Z$ introduces $\sum_{k=1}^{m} \sum_{j \in \operatorname{ch}(k)} 2\left|\eta_{j}\right|\left(2\left|\eta_{j}\right|+1\right) / 2$ equality constraints, which is more than twice the number necessary when conversion is applied to $X$.

The conversion method can be applied to a cone LP of the form (4). However, since the conversion method only affects the positive semidefinite matrix variables, we will simplify our notation by considering the conversion method applied to a semidefinite optimization problem of the form

$$
\begin{array}{ll}
\operatorname{minimize} & \operatorname{tr}\left(A_{0} X\right) \\
\text { subject to } & \operatorname{tr}\left(A_{j} X\right)=b_{j}, j=1, \ldots, r, \\
& X \in \mathcal{H}_{E, \mathrm{c}}^{n}
\end{array}
$$

where $A_{j} \in \mathcal{H}_{E}^{n}, j=0,1, \ldots, r$. Notice that we can express the inner products $\operatorname{tr}\left(A_{j} X\right)$ in terms of $W_{1}, \ldots, W_{m}$ as

$$
\operatorname{tr}\left(A_{j} X\right)=\sum_{k=1}^{m} \operatorname{tr}\left(\widetilde{A}_{j, k} W_{k}\right)
$$

where the matrices $\widetilde{A}_{j, k} \in \mathcal{H}^{\left|\gamma_{k}\right|}$ must satisfy the condition $A_{j}=\sum_{k=1}^{m} \mathcal{E}^{\operatorname{adj}}\left(\widetilde{A}_{j, k}\right)$. In general there are infinitely many ways of splitting $A_{j}$ in this way.

The conversion of (7) is a semidefinite optimization problem with $m$ blocks, i.e.,

$$
\begin{array}{cl}
\operatorname{minimize} & \sum_{k=1}^{m} \operatorname{tr}\left(\widetilde{A}_{0, k} W_{k}\right) \\
\text { subject to } & \sum_{k=1}^{m} \operatorname{tr}\left(\widetilde{A}_{j, k} W_{k}\right)=b_{j}, j=1, \ldots, r, \\
& \text { for } k=1, \ldots, m \text { and } j \in \operatorname{ch}(k), \\
& \mathcal{E}_{\gamma_{j} \cap \gamma_{k}}\left(\mathcal{E}_{\gamma_{j}}^{\operatorname{adj}}\left(W_{j}\right)-\mathcal{E}_{\gamma_{k}}^{\operatorname{adj}}\left(W_{k}\right)\right)=0 \\
& W_{k} \succeq 0, k=1, \ldots, m .
\end{array}
$$

The total number of equality constraints is $r+s$ where $s$ is the number of consistency constraints. For example, if the sparsity graph is an acyclic graph, then there are $m=n-1$ cliques of order 2 and $s=n-1$ consistency constraints. Thus, in this special case, the SDR is equivalent to the SOC relaxation obtained by enforcing $n-1$ principal minors of $X$ to be positive semidefinite and expressing these constraints as SOC constraints. For general problems, we will see in Section $\mathrm{V}$ that when applying the conversion method directly to the cone LP (4), the number of consistency constraints $s$ may be several times larger than $r$. However, the number of variables in the converted problem is typically much smaller than in the unconverted problem since the $m$ blocks are equivalent to $\sum_{i=1}^{m}\left|\gamma_{i}\right|^{2}$ scalar real-valued variables instead of $|\mathcal{N}|^{2}$.

As mentioned in the introduction, the computational bottleneck when solving an SDP is typically forming and factorizing the Schur complement equations that define the search direction at each interior-point iteration. The Schur complement matrix $H$ associated with (7) is of order $r$ with entries of the form $H_{k l}=\operatorname{tr}\left(A_{k} S^{-1} A_{l} S^{-1}\right)$ for some $S \in \mathcal{H}_{E,+}^{n}$, and hence $H$ is generally dense. The Schur complement system associated with (9), however, is often sparse, but it is of order $r+s$ instead of $r$. A detailed exposition pertaining to conversion and sparsity in the Schur complement system is outside the scope of this paper; see e.g. [27], [28] and references therein.

\section{Clique Amalgamation}

The conversion method introduces a large number of equality constraints when some of the sets $\eta_{j}$ are large. Amalgamating (i.e., merging) a clique $j$ and its parent in the clique tree (say, clique $k$ ), reduces the number of cliques by one, and it also reduces the number of equality constraints by $\left|\eta_{j}\right|^{2}$. This, in turn, implies that the order of the Schur complement system is reduced, but it also affects the sparsity of the Schur complement system. Moreover, the new combined clique is given by $\gamma_{k} \cup \gamma_{j}$, and hence it is larger than both of the cliques from which it was constructed. Thus, there are two different but coupled trade-offs to consider, namely a trade-off between the number of cliques and their order (many small cliques or fewer but larger cliques) and a trade-off between the order of the Schur complement system and its sparsity (a large sparse system or a smaller but less sparse system).

In this paper, we will use the greedy clique amalgamation heuristic from Sun et al. [28] which does not take the sparsity of the Schur complement system into account. Specifically, we start at the bottom of the tree and merge clique $j$ and its parent clique $k$ if $\left(\left|\gamma_{k}\right|-\left|\eta_{j}\right|\right)\left(\left|\gamma_{j}\right|-\left|\eta_{j}\right|\right) \leq t_{\text {fill }}$ or $\max \left(\left|\gamma_{j}\right|-\right.$ $\left.\left|\eta_{j}\right|,\left|\gamma_{k}\right|-\left|\eta_{k}\right|\right) \leq t_{\text {size }}$. Here $t_{\text {fill }}$ is a threshold based on the amount of fill induced by merging clique $j$ and its parent, and $t_{\text {size }}$ is a threshold based on the cardinality of the so-called supernodes which are the sets $\gamma_{k} \backslash \eta_{k}$ and $\gamma_{j} \backslash \eta_{j}$.

We end this section by noting that the term "clique amalgamation" is inspired by terminology from the sparse factorization literature where "supernodal amalgamation" refers to a similar technique used to balance the number of supernodes and their orders to obtain cache-efficient block sizes [29], [30].

\section{Conversion-BAsed SEmidefinite Relaxation}

In this section, we propose a new SDR technique for sparse nonconvex QPs of the form

$$
\begin{array}{ll}
\operatorname{minimize} & x^{H} A_{0} x \\
\text { subject to } & x^{H} A_{j} x=b_{j}, j=1, \ldots, r,
\end{array}
$$

where $A_{0}, A_{1}, \ldots, A_{r} \in \mathcal{H}_{E}^{n}$ are given and $E$ is chordal with cliques $\gamma_{1}, \ldots, \gamma_{m}$. An SDR of (10) is given by (7) where $X \in \mathcal{H}_{E, \mathrm{c}}^{n}$ is a convex relaxation of the constraint $X=x x^{H}$. Recall that applying the conversion method to (7) yields the equivalent problem (9). We refer to (9) as "full conversion" which is closely related to the sparse SDR technique of Waki et al. [31] for polynomial optimization with structured sparsity.

Now recall that the consistency constraint (6) that couples clique $j$ and its parent clique is equivalent to $\left|\eta_{j}\right|^{2}$ equality 
constraints. By dropping some of these equality constraints, we can obtain computationally cheaper relaxations. We will refer to this method as "conversion-based SDR" (CSDR), and we will see in Section $\mathrm{V}$ that $O\left(\left|\eta_{j}\right|\right)$ equality constraints linking each clique $j$ and its parent are often sufficient to recover the solution to the standard SDR. This is not surprising since we can verify whether two Hermitian rank-1 matrices of order $\left|\eta_{j}\right|$ are equal or not by comparing a single column or row. This corresponds to $2\left|\eta_{j}\right|-1$ equality constraints since each row/column contains $\left|\eta_{j}\right|$ real values and $\left|\eta_{j}\right|-1$ complex values. Note that the CSDR solution is also a solution to the standard SDR whenever it satisfies the full set of consistency constraints (6). In the remainder of this section, we discuss CSDRs based on different heuristic consistency strategies.

\section{A. Band CSDR}

A straightforward method for reducing the number of consistency equalities is to keep only equalities that correspond to entries of $\mathcal{E}_{\gamma_{j} \cap \gamma_{k}}\left(\mathcal{E}_{\gamma_{j}}^{\operatorname{adj}}\left(W_{j}\right)-\mathcal{E}_{\gamma_{k}}^{\operatorname{adj}}\left(W_{k}\right)\right)=0$ within a band of half-bandwidth $\rho_{j}$ (where $0 \leq \rho_{j}<\left|\eta_{j}\right|$ ). Diagonal consistency corresponds to $\rho_{j}=0$, and full consistency corresponds to $\rho_{j}=\left|\eta_{j}\right|-1$. To simplify the choice of parameters, we define a "global" half-bandwidth parameter $\rho \geq 0$ and let $\rho_{j}=\min \left(\rho,\left|\eta_{j}\right|-1\right)$. The total number of consistency constraints in the band CSDR is then given by

$$
\sum_{k=1}^{m} \sum_{j \in \operatorname{ch}(k)}\left(\left|\eta_{j}\right|+2 \sum_{l=1}^{\rho_{j}}\left(\left|\eta_{j}\right|-l\right)\right) .
$$

Recall that $\rho=0$ corresponds to diagonal-only coupling, and this is generally not sufficient to recover a feasible solution to the original problem even if the CSDR solution has rank1 blocks. This is because the diagonal elements of a rank-1 block correspond to squared voltage magnitudes, and hence phase consistency is not enforced. However, for $\rho \geq 1$, we can always recover a solution to the original problem if all blocks have rank 1 since the additional equality constraints are sufficient to recover relative phase information.

\section{B. Other CSDRs}

An alternative to band CSDR is to keep only equality constraints that correspond to diagonal entries as well as the entries in $\rho_{j}=\min \left(\rho,\left|\eta_{j}\right|-1\right)$ rows/columns with $\rho \geq 0$ (e.g., an arrow pattern). This approach leads to exactly the same number of equality constraints as in the band CSDR with parameter $\rho$, and we can recover a solution to the original problem if $\rho \geq 1$ and all blocks have rank 1 .

Yet another possibility is to keep equality constraints that correspond to nonzero entries in the bus admittance matrix (i.e., corresponding to edges in the network graph). The total number of consistency equalities is then equal to $\sum_{k=1}^{m} \sum_{j \in \operatorname{ch}(k)}\left(\left|\eta_{j}\right|+2\left|\mathcal{L}_{j}\right|\right)$ where $\mathcal{L}_{j} \subseteq \mathcal{L}$ is the subset of transmission lines that connect a pair of power busses that each belong to both clique $j$ and its parent clique. We refer to this strategy as sparse CSDR. It is also possible to combine several consistency strategies, for example, using band-plussparse structure.

\begin{tabular}{lrrrrrr}
\hline Case & $|\mathcal{N}|$ & $\left|\mathcal{G}^{\text {fix }}\right|$ & $\left|\mathcal{G}^{\text {lin }}\right|$ & $\left|\mathcal{G}^{\text {quad }}\right|$ & $|\mathcal{L}|$ & $|\mathcal{F}|$ \\
\hline IEEE-118 & 118 & 0 & 0 & 54 & 186 & 0 \\
IEEE-300 & 300 & 0 & 0 & 69 & 409 & 0 \\
2383wp & 2,383 & 92 & 235 & 0 & 2,896 & 5 \\
2736sp & 2,736 & 118 & 82 & 0 & 3,269 & 1 \\
2737sp & 2,737 & 165 & 54 & 0 & 3,269 & 1 \\
2746wop & 2,746 & 346 & 85 & 0 & 3,307 & 0 \\
2746wp & 2,746 & 352 & 104 & 0 & 3,279 & 0 \\
3012wp & 3,012 & 9 & 376 & 0 & 3,572 & 5 \\
3120sp & 3,120 & 25 & 273 & 0 & 3,693 & 8 \\
\hline \multicolumn{7}{c}{ TABLE I } \\
\multicolumn{7}{c}{ TEST CASES AND PROBLEM DIMENSIONS }
\end{tabular}

As a final remark, we mention the possibility to use the conversion technique based on the cliques of a nonchordal embedding of the network graph (or the network graph itself) instead of the cliques of a chordal embedding. Positive semidefiniteness of the cliques of a partial Hermitian matrix is a necessary condition for it to have a positive semidefinite completion. However, we know from the theorem of Grone et al. [25, Theorem 7] that this is not a sufficient condition in general unless the sparsity pattern is chordal. Hence by applying the conversion method based on the cliques of a nonchordal patterns, we can obtain SDRs that are computationally cheaper to solve (but generally also weaker) than the standard SDR. We will not explore this strategy further in this paper.

\section{NUMERICAL EXPERIMENTS}

We have implemented and tested the SDR of the problem in (3) as well as some of the conversion-based SDR techniques from Section IV. The experiments are based on the benchmark problems from the MATPOWER package [17], and we build the data matrices associated with the cone LP (4) directly without using a modeling tool. The experiments were carried out in Matlab R2013a on a laptop with an Intel Core i5 dual-core 1.8 GHz CPU and $8 \mathrm{~GB}$ RAM, and we used SeDuMi 1.3 with tolerance $\epsilon=10^{-7}$ to solve the cone LP (4) and its different conversions. Note that although we explicitly build the complex-valued cone LP (4) and apply the conversion method to this formulation, we cast the converted problem as a real-valued cone LP before passing it to SeDuMi.

Following the approach in [16], we treat generators with tight upper and lower bounds as generators with fixed power. Specifically, we set $p_{g}=\left(P_{g}^{\min }+P_{g}^{\max }\right) / 2$ if $P_{g}^{\max }-P_{g}^{\min }$ is less than 0.001 units for generator $g$, and $\mathcal{G}^{\text {fix }}$ denotes the set of number of such generators. We also eliminate transmission line flow constraints that are not active (i.e., operating below their maximum capacity) at the (local) solution provided by MATPOWER. (For the test problems with several thousands of power busses, SeDuMi did not return a useful solution when all the transmission line constraints were included in the problem.) Table I lists the test cases along with relevant problem dimensions. The number of active transmission line constraints are listed in the last column of the table.

In order to improve the conditioning of the problem, we scale the problem data $c, G$, and $h$ in the cone LP formulation (4) as $G:=G D^{-1}, c:=D^{-1} c$, and $h:=h /\|h\|_{2}$ where $D=\operatorname{diag}\left(d_{1}, d_{2}, \ldots, d_{r+s}\right)$ and $d_{k}=\max \left(\left|c_{k}\right|,\left\|G e_{k}\right\|_{\infty}\right)$. 


\begin{tabular}{|c|c|c|c|c|c|c|c|}
\hline \multirow[t]{2}{*}{ Case } & \multirow{2}{*}{$\begin{array}{r}\text { \# constraints } \\
\text { (unconv.) }\end{array}$} & \multirow{2}{*}{$\begin{array}{l}\text { Full } \\
\text { conv. }\end{array}$} & \multirow{2}{*}{$\begin{array}{l}\text { Amal. } \\
\text { conv. }\end{array}$} & \multicolumn{3}{|c|}{ Band CSDR $(\rho)$} & \multirow{2}{*}{$\begin{array}{l}\text { Sparse } \\
\text { CSDR }\end{array}$} \\
\hline & & & & 1 & 2 & 3 & \\
\hline IEEE-118 & $742 \times 1.0$ & 1.78 & 1.10 & 1.03 & 1.07 & 1.09 & 1.04 \\
\hline IEEE-300 & $1,545 \times 1.0$ & 1.99 & 1.14 & 1.04 & 1.09 & 1.12 & 1.04 \\
\hline 2383wp & $10,000 \times 1.0$ & 2.97 & 1.63 & 1.08 & 1.20 & 1.30 & 1.10 \\
\hline 2736sp & $11,248 \times 1.0$ & 3.04 & 1.64 & 1.08 & 1.22 & 1.32 & 1.10 \\
\hline $2737 \mathrm{sp}$ & $11,163 \times 1.0$ & 3.03 & 1.62 & 1.08 & 1.22 & 1.32 & 1.10 \\
\hline 2746wop & $11,379 \times 1.0$ & 3.10 & 1.63 & 1.08 & 1.21 & 1.32 & 1.10 \\
\hline 2746wp & $11,438 \times 1.0$ & 3.00 & 1.62 & 1.08 & 1.21 & 1.32 & 1.09 \\
\hline $3012 w p$ & $12,716 \times 1.0$ & 3.03 & 1.67 & 1.08 & 1.22 & 1.32 & 1.09 \\
\hline 3120 sp & $12,990 \times 1.0$ & 3.10 & 1.72 & 1.08 & 1.22 & 1.33 & 1.09 \\
\hline
\end{tabular}

NUMBER OF CONSTRAINTS BEFORE AND AFTER CONVERSION

\begin{tabular}{lcccccc}
\hline Case & Full & Amal. & \multicolumn{3}{c}{ Band CSDR $(\rho)$} & Sparse \\
\cline { 4 - 6 } & conv. & conv. & 1 & 2 & 3 & CSDR \\
\hline IEEE-118 & $1.5 \mathrm{E}+6$ & $1.1 \mathrm{E}+7$ & $1.1 \mathrm{E}+1$ & $2.0 \mathrm{E}+7$ & $1.9 \mathrm{E}+7$ & $1.1 \mathrm{E}+1$ \\
IEEE-300 & $2.4 \mathrm{E}+2$ & $1.2 \mathrm{E}+3$ & $1.7 \mathrm{E}-1$ & $1.2 \mathrm{E}+3$ & $1.1 \mathrm{E}+3$ & $1.7 \mathrm{E}-1$ \\
$2383 w p$ & $4.0 \mathrm{E}+2$ & $5.3 \mathrm{E}+2$ & $3.7 \mathrm{E}-1$ & $9.4 \mathrm{E}+1$ & $3.8 \mathrm{E}+2$ & $4.1 \mathrm{E}-1$ \\
$2736 \mathrm{sp}$ & $1.6 \mathrm{E}+5$ & $5.9 \mathrm{E}+5$ & $1.2 \mathrm{E}-1$ & $4.0 \mathrm{E}+2$ & $1.1 \mathrm{E}+5$ & $1.2 \mathrm{E}-1$ \\
2737sp & $1.8 \mathrm{E}+4$ & $7.2 \mathrm{E}+4$ & $1.2 \mathrm{E}-1$ & $6.2 \mathrm{E}+2$ & $2.8 \mathrm{E}+4$ & $1.2 \mathrm{E}-1$ \\
2746wop & $2.0 \mathrm{E}+4$ & $5.6 \mathrm{E}+4$ & $1.5 \mathrm{E}-1$ & $1.2 \mathrm{E}+2$ & $3.2 \mathrm{E}+4$ & $4.8 \mathrm{E}-1$ \\
2746wp & $3.5 \mathrm{E}+5$ & $3.9 \mathrm{E}+5$ & $1.3 \mathrm{E}-1$ & $3.9 \mathrm{E}+2$ & $1.9 \mathrm{E}+5$ & $1.3 \mathrm{E}-1$ \\
$3012 \mathrm{wp}$ & $8.5 \mathrm{E}+0$ & $1.6 \mathrm{E}+2$ & $2.4 \mathrm{E}-1$ & $7.5 \mathrm{E}+1$ & $2.2 \mathrm{E}+2$ & $2.4 \mathrm{E}-1$ \\
$3120 \mathrm{sp}$ & $6.6 \mathrm{E}+1$ & $1.5 \mathrm{E}+2$ & $2.8 \mathrm{E}-1$ & $6.9 \mathrm{E}+1$ & $1.6 \mathrm{E}+2$ & $2.8 \mathrm{E}-1$
\end{tabular}

TABLE III

EIGENVALUE RATIOS

This scales the dual variables and the objective, but not the primal variables. We found that this scaling heuristic reduced the number of iterations and improved the solution accuracy in most of the large-scale problems. Like in [8], we also use a minimum line resistance of $10^{-4}$ per unit in our experiments.

For each test case, we solve the following SDRs: SDR with full conversion based on chordal embedding with/without clique amalgamation, band CSDR (with clique amalgamation), and sparse CSDR (also with clique amalgamation). We use the clique amalgamation parameters $t_{\text {size }}=t_{\text {fill }}=16$. Table II lists the number of constraints in each of the SDR problems. The second column lists the number of constraints $r$ in the unconverted problem, and columns 3-8 list $(r+s) / r$ which is the total number of constraints normalized by the number of constraints in the unconverted problem. Recall that the "full" and "amalgamated" conversions are equivalent to the unconverted problem whereas the "band" and "sparse" CSDRs are weaker, but computationally cheaper, relaxations. The full conversion method adds the most equality constraints, and for the large problems, this approach roughly triples the number of constraints. The amalgamated conversion method is clearly much more economical in term of the number of added equality constraints, but the number of constraints still grows with more than $60 \%$ when converting large problems. The CSDRs introduce the smallest number of constraints, i.e., around 10\%-30\% for large problems, depending on the value of $\rho$. As a result, and as we will see later in this section, the CSDRs are often much cheaper to solve.

The ratio between the largest and the second largest eigenvalue can be used as an indicator for the numerical rank of the solution. Roughly speaking, the solution has numerical rank 1 if the aforementioned eigenvalue ratio is sufficiently

\begin{tabular}{lcccc}
\hline \multirow{2}{*}{ Case } & \multicolumn{3}{c}{ Band CSDR $(\rho)$} & Sparse \\
\cline { 2 - 4 } & 1 & 2 & 3 & CSDR \\
\hline IEEE-118 & 0.999 & 1.000 & 1.000 & 0.999 \\
IEEE-300 & 0.999 & 1.000 & 1.000 & 0.999 \\
2383wp & 0.990 & 0.998 & 1.000 & 0.990 \\
2736sp & 0.989 & 1.000 & 1.000 & 0.990 \\
2737sp & 0.980 & 1.000 & 1.000 & 0.979 \\
2746wop & 0.978 & 0.996 & 1.000 & 0.978 \\
2746wp & 0.989 & 1.000 & 1.000 & 0.989 \\
3012wp & 0.985 & 0.994 & 0.998 & 0.985 \\
3120sp & 0.988 & 0.999 & 1.000 & 0.989 \\
\hline \multicolumn{4}{c}{ TABLE IV }
\end{tabular}

NORMALIZED OBJECTIVE VALUE FOR CSDRS

\begin{tabular}{lrrrrrrrrr}
\hline Case & No & Full & Amal. & \multicolumn{7}{c}{ Band CSDR $(\rho)$} & Sparse & {$[16]$} \\
& conv. & conv. & conv. & \multicolumn{1}{c}{1} & 2 & 3 & & CSDR & \\
\hline IEEE-118 & 5.4 & 7.3 & 2.2 & 1.2 & 1.5 & 2.1 & 1.2 & 2.1 \\
IEEE-300 & 78 & 19 & 5.0 & 3.8 & 4.2 & 4.0 & 3.7 & 5.7 \\
2383wp & - & 650 & 225 & 78 & 103 & 132 & 85 & 730 \\
2736sp & - & 484 & 145 & 56 & 72 & 102 & 74 & 622 \\
2737sp & - & 716 & 200 & 57 & 107 & 133 & 93 & 607 \\
2746wop & - & 439 & 138 & 51 & 104 & 86 & 65 & 738 \\
2746wp & - & 547 & 168 & 50 & 95 & 95 & 82 & 752 \\
3012wp & - & 575 & 201 & 57 & 87 & 86 & 78 & 1197 \\
$3120 \mathrm{sp}$ & - & 718 & 217 & 57 & 85 & 96 & 96 & 1619 \\
\hline \multicolumn{8}{c}{ TABLE V } \\
\hline
\end{tabular}

large. Since the converted problems have multiple blocks, we consider the smallest such ratio, and these are listed in Table III. It is interesting to note that full conversion with clique amalgamation yields slightly better results than without. In particular, for the problem $3012 \mathrm{wp}$, there is an order of magnitude difference between the eigenvalue ratio for the two methods. Furthermore, for the band CSDR, the eigenvalue ratio improves when the half-bandwidth $\rho$ is increased, and for $\rho=3$, the eigenvalue ratios are comparable to those obtained via full conversion. The sparse CSDR yields results that are similar to the band CSDR with $\rho=1$, and despite the lack of a rank-1 solution, the nearest rank-1 approximation may still be useful as initialization for a general nonlinear solver.

Recall that in general, the CSDRs are weaker than the standard SDR. This implies that the objective value can be used as an indication of the relaxation quality. Table IV lists the objective values obtained via the CSDRs, normalized by the objective values obtained via the standard SDR. A normalized objective value of 1 corresponds to a relaxation that is as tight as the original SDR. Notice that the CSDRs all yield lower bounds that are within a few percent of the objective value obtained via the standard SDR, and in all but one case, the band CSDR with $\rho=3$ yields a solution of the same quality as that obtained via the standard SDR.

Finally we compare the computational complexity in terms of computation time required to solve each of the relaxations. Table V shows "wall time" in seconds as reported by SeDuMi (the "CPU time" was roughly a factor of two larger for all problems). If we compare the full conversion and the amalgamated conversion, we see that clique amalgamation typically results in a speed-up of around 2.5-3.5. Furthermore, the time required to solve the band CSDR with $\rho=3$ is 
roughly 100 seconds for the large-scale problems which is a speed-up of around 5-6 when compared to the time required to solve the full conversion SDR. The last column in the table lists the results reported in [16], and although a direct comparison is difficult (the experiments were performed on different machines and using different formulations), the large margins suggest that our approach is competitive and often much faster than previously proposed methods.

\section{CONCLUSIONS}

We have proposed a new method for generating computationally cheaper SDRs of AC OPF problems. The method is based on chordal conversion, which, given a chordal embedding of the network graph, converts a sparse semidefinite optimization problem of the form (7) into an equivalent block-diagonal problem (9) that includes a set of consistency constraints. By including only a subset of the consistency constraints, we can generate conversion-based SDRs with reduced computational cost, but these SDRs may also be weaker than the standard SDR. The band CSDR method from Section IV keeps only consistency equalities associated with entries within a band of half-bandwidth $\rho$, and our numerical experiments indicate that this strategy works surprisingly well in practice. More specifically, the band CSDR with $\rho=3$ has the same objective value as the standard SDR in all but one test case, and the weaker band CSDRs yield objective values that are within a few percent of those obtained via the standard SDR. However, the experiments are based on only a small number of test cases, so further experiments are necessary to thoroughly evaluate the quality of the CSDRs.

In addition to the complexity-reducing CSDR technique, we lower the computational cost further by applying the conversion method to the complex-valued problem instead of its real-valued counterpart. Moreover, we model transmission line flow constraints and generators with quadratic fuel cost using SOC constraints, which reduces the total number of variables and therefore also computational cost. By combining these techniques, we have shown that it is possible to solve SDRs of large-scale OPF problems significantly faster than with previously proposed methods.

The CSDR technique can also be applied to extensions of the OPF formulation (3). One such extension is the socalled multi-period OPF where power generation and demand vary over time, and the time slots are coupled because of generator ramp rate limits. With this kind of formulation, the standard SDR has a Hermitian positive semidefinite matrix $X^{(i)}$ of order $|\mathcal{N}|$ for each time slot $i$, and hence the problem dimension grows quickly with the number of time periods. The CSDR technique can therefore be expected to provide significant computational savings when applied to the SDR of the multi-period OPF problem.

\section{REFERENCES}

[1] J. Carpentier, "Contribution à l'étude du dispatching économique," Bulletin de la Société française des électriciens, vol. 8, no. 3, pp. 431447, 1962

[2] J. A. Momoh, Electric Power System Applications of Optimization. Marcel Dekker, 2001
[3] J. Zhu, Optimization of Power System Operation. Wiley, 2009.

[4] Z. Qiu, G. Deconinck, and R. Belmans, "A literature survey of optimal power flow problems in the electricity market context," in Power Systems Conference and Exposition, 2009.

[5] R. D. Zimmerman, C. E. Murillo-Sánchez, and R. J. Thomas, "MATPOWER steady-state operations, planning and analysis tools for power systems research and education," IEEE Trans. Power Syst., vol. 26, no. 1, pp. 12-19, 2011.

[6] X. Bai, H. Wei, K. Fujisawa, and Y. Wang, "Semidefinite programming for optimal power flow problems," International Journal of Electrical Power \& Energy Systems, vol. 30, no. 6, pp. 383-392, 2008.

[7] J. Lavaei and S. H. Low, "Convexification of optimal power flow problem," in Proc. of the 48th Annual Allerton Conference on Communication, Control, and Computing, 2010, pp. 223-232.

[8] — "Zero duality gap in optimal power flow problem," IEEE Trans Power Syst., vol. 27, no. 1, pp. 92-107, 2012.

[9] S. Bose, D. F. Gayme, S. Low, and K. M. Chandy, "Optimal power flow over tree networks," in Proc. of the 49th Annual Allerton Conference on Communication, Control, and Computing, 2011, pp. 1342-1348.

[10] B. Zhang and D. Tse, "Geometry of injection regions of power networks," IEEE Trans. Power Syst., vol. 28, no. 2, pp. 788-797, 2013.

[11] J. F. Sturm, "Using SEDUMI 1.02, a Matlab toolbox for optimization over symmetric cones," Optimization Methods and Software, vol. 11-12, pp. 625-653, 1999.

[12] S. J. Benson and Y. Ye, "DSDP5: A software package implementing the dual-scaling algorithm for semidefinite programming," Mathematics and Computer Science Division, Argonne National Laboratory, Tech. Rep. ANL/MCS-TM-255, 2004.

[13] M. S. Andersen, J. Dahl, and L. Vandenberghe, "Implementation of nonsymmetric interior-point methods for linear optimization over sparse matrix cones," Math. Prog. Computation, vol. 2, pp. 167-201, 2010.

[14] R. A. Jabr, "Exploiting sparsity in SDP relaxations of the OPF problem," IEEE Trans. Power Syst., vol. 27, no. 2, pp. 1138-1139, May 2012.

[15] M. Fukuda, M. Kojima, K. Murota, and K. Nakata, "Exploiting sparsity in semidefinite programming via matrix completion I: general framework," SIAM Journal on Optimization, vol. 11, pp. 647-674, 2000.

[16] D. K. Molzahn, J. T. Holzer, B. C. Lesieutre, and C. L. DeMarco, "Implementation of a large-scale optimal power flow solver based on semidefinite programming," IEEE Trans. Power Syst., vol. PP, no. 99, pp. 1-12, 2013.

[17] R. D. Zimmerman and C. E. Murillo-Sánchez, "MATPOWER 4.1 User's Manual," Dec. 2011.

[18] A. Ben-Tal and A. Nemirovski, Lectures on Modern Convex Optimization. Analysis, Algorithms, and Engineering Applications. SIAM, 2001.

[19] S. Kim and M. Kojima, "Second order cone programming relaxation of nonconvex quadratic optimization problems," Optimization Methods and Software, vol. 15, no. 3-4, pp. 201-224, 2001.

[20] J. R. S. Blair and B. Peyton, "An introduction to chordal graphs and clique trees," in Graph Theory and Sparse Matrix Computation, A. George, J. R. Gilbert, and J. W. H. Liu, Eds. Springer-Verlag, 1993.

[21] M. C. Golumbic, Algorithmic Graph Theory and Perfect Graphs, 2nd ed. Elsevier, 2004.

[22] M. S. Andersen, "Chordal sparsity in interior-point methods for conic optimization," Ph.D. dissertation, University of California, Los Angeles, 2011.

[23] A. Pothen and C. Sun, "Compact clique tree data structures in sparse matrix factorizations," in Large-Scale Numerical Optimization, T. F. Coleman and Y. Li, Eds. SIAM, 1990, pp. 180-204.

[24] M. S. Andersen, J. Dahl, and L. Vandenberghe, "Logarithmic barriers for sparse matrix cones," Optimization Methods and Software, vol. 28, no. 3, pp. 396-423, 2013.

[25] R. Grone, C. R. Johnson, E. M. Sá, and H. Wolkowicz, "Positive definite completions of partial Hermitian matrices," Linear Algebra and Appl., vol. 58, pp. 109-124, 1984

[26] S. Kim, M. Kojima, M. Mevissen, and M. Yamashita, "Exploiting sparsity in linear and nonlinear matrix inequalities via positive semidefinite matrix completion," Math. Prog., vol. 129, pp. 33-68, 2011.

[27] K. Kobayashi, S. Kim, and M. Kojima, "Correlative sparsity in primaldual interior-point methods for LP, SDP, and SOCP," Applied Mathematics and Optimization, vol. 58, no. 1, pp. 69-88, 2008.

[28] Y. Sun, M. S. Andersen, and L. Vandenberghe, "Decomposition in conic optimization with partially separable structure," arXiv:1306.0057, 2013.

[29] C. Ashcraft and R. Grimes, "The influence of relaxed supernode partitions on the multifrontal method," ACM Trans. on Mathematical Software, vol. 15, no. 4, pp. 291-309, 1989.

[30] J. Hogg and J. Scott, "A modern analyse phase for sparse tree-based direct methods," Rutherford Appleton Laboratory, Tech. Rep., 2010. 
[31] H. Waki, S. Kim, M. Kojima, and M. Muramatsu, "Sums of squares and semidefinite program relaxations for polynomial optimization problems with structured sparsity," SIAM Journal on Optimization, vol. 17, no. 1, pp. 218-241, 2006.

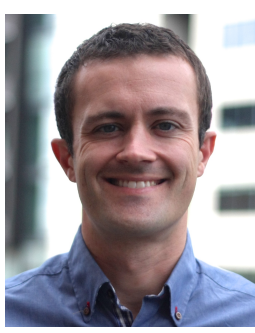

Martin S. Andersen received his M.S. in Electrical Engineering from Aalborg University, Denmark (2006), and his Ph.D. in Electrical Engineering from the University of California, Los Angeles (2011). He was a postdoctoral researcher from 2011 until 2012 in the Division of Automatic Control at Linköping University, Sweden, and he is currently a postdoctoral researcher at the Technical University of Denmark in the Department of Applied Mathematics and Computer Science. His research interests include processing, systems and control. optimization, numerical methods, signal and image

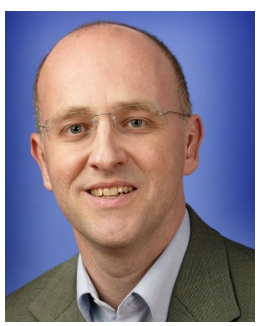

Anders Hansson (M'97, SM'09) received the Master of Science degree in Electrical Engineering in 1989, the Degree of Licentiate of Engineering in Automatic Control in 1991, and the PhD in Automatic Control in 1995, all from Lund University, Sweden. In 1989, 1992, and 1995, he spent three months at Landis \& Gyr AG, Switzerland. During the academic year 1992-1993, he spent six months at Imperial College in London, UK. From 1995 until 1997, he was a postdoctoral student, and from 1997 until 1998, a research associate at the Information Systems Lab, Department of Electrical Engineering, Stanford University. In 1998, he was appointed assistant professor, and in 2000, associate professor (docent) at S3-Automatic Control, Royal Institute of Technology, Stockholm, Sweden. He was appointed associate professor in 2001 at the Division of Automatic Control, Linkping University, and since 2006, he has been full professor in the same department. During the academic year 2011-2012, he was a Visiting Professor at the University of California, Los Angeles.

Dr. Hansson was an associate editor of the IEEE Transactions on Automatic Control from 2006-2007. He is a member of the EUCA Council (since 2009), of the Technical Committee on Systems with Uncertainty of the IEEE Control Systems Society (since 2009), and of the IFAC Technical Committee on Robust Control (since 2012). His research interests are within the fields of optimal control, stochastic control, linear systems, signal processing, fuzzy logic, applications of control, image processing, and telecommunications. $\mathrm{He}$ got the SAAB-Scania Research Award in 1992.

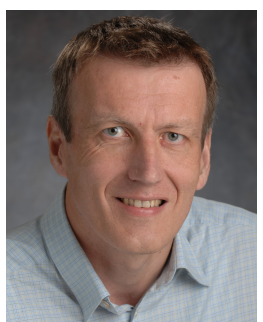

Lieven Vandenberghe (M97, SM'08) received the Electrical Engineering degree and the Ph.D. degree in electrical engineering from the Katholieke Universiteit Leuven (K.U. Leuven), Belgium, in 1987 and 1992, respectively. He is currently a Professor in the Electrical Engineering Department and the Department of Mathematics at the University of California, Los Angeles (UCLA). He joined UCLA in 1997 as an Assistant Professor, following postdoctoral appointments at K.U. Leuven and Stanford University. He has held visiting Professor positions at K.U. Leuven and the Technical University of Denmark.

Dr. Vandenberghe is author (with S. Boyd) of the book Convex Optimization (Cambridge University Press, 2004), and editor (with H. Wolkowicz and R. Saigal) of the volume Handbook of Semidefinite Programming (Kluwer, 2000). His main research interests are in optimization, systems and control, and signal processing. He received the IEEE Guillemin-Cauer Award in 1990, the Robert Stock Award for Exact and Biomedical Sciences at K.U. Leuven in 1993, an NSF CAREER Award in 1998, and the TRW Excellence in Teaching Award of the UCLA Henry Samueli School of Engineering and Applied Science in 2002. 